\title{
A influência do número de barras na resistência à compressão axial dos fixadores externos monoplanares em modelos de tíbias de poliuretano
}

\author{
The influence of the number of bars on the axial compressive strength of \\ uniplanar external fixators in polyurethane tibia models
}

\author{
Márcio Hiroaki Kume ${ }^{1}$; Osvaldo Malafaia, ECBC-PR²; Ulrich Alexandre Dietz'; Mark Deeke ${ }^{3}$; Karin Soldatelli Borsato ${ }^{4}$; Flavia \\ Gonçalves Menegotto ${ }^{5}$; Marcia Regina Zanello Pundek ${ }^{5}$
}

\section{RE S U M O}

\begin{abstract}
Objetivo: Analisar a resistência (rigidez) do sistema de fixação externa tubular uniplanar, com hastes de conexão única e dupla, com traços de fraturas estáveis e instáveis. Métodos: Foram utilizados 48 modelos semelhantes à tíbia. Em todos foi deixado um intervalo de $0,5 \mathrm{~cm}$ entre os fragmentos e realizados cortes com angulações de $15^{\circ}$ e $45^{\circ}$ para simular fraturas estáveis e instáveis, respectivamente. Os modelos foram divididos em quatro grupos de acordo com o traço fraturário $\left(15^{\circ}\right.$ e $\left.45^{\circ}\right)$ e o número de barras metálicas na montagem (1 e 2 barras). Os modelos de prova foram adaptados à uma máquina de testes Instron ${ }^{\circledR}$, pelas suas extremidades, e submetidos à compressão axial até que os fragmentos tiveram contato total. Avaliou-se a força necessária para efetuar o completo contato dos fragmentos do modelo. Resultados: As forças instabilizadoras na montagem do fixador com barra dupla foram bastante superiores às com barra única. Observou-se ainda que o grupo com barra única instável apresentou variabilidade muito menor que os demais grupos, ou seja, apresenta resultados mais homogêneos, além de ter apresentado a menor média. Conclusão: A montagem do fixador externo com uma haste longitudinal dupla nos modelos estudados é mais estável que as demais quando submetidas à uma força de compressão axial.
\end{abstract}

Descritores: Fixadores externos. Tíbia.

\section{INTRODUÇÃO}

$\mathrm{N}$ estabilização cirúrgica de fraturas, o objetivo principal consiste em obter-se condições consideradas ótimas para a cicatrização dos tecidos, evitando complicações e otimizando a reabilitação do paciente. $\mathrm{O}$ uso dos dispositivos, chamados fixadores externos, popularizou-se no tratamento das enfermidades ósseas, em especial no das fraturas dos membros inferiores. Amplamente aplicados no tratamento das fraturas expostas da tíbia ${ }^{1}$ e para os alongamentos ósseos, seu uso é conhecido há muito tempo, na história da medicina, como relatado por Sisk². Conceituam-se os fixadores externos como aparelhos, geralmente manufaturados em metal, compostos por pinos rosqueados, conectores e hastes de suportes longitudinais, aplicados por técnicas percutâneas, com o intuito de estabilizar um osso ou uma articulação. O estudo de cada elemento faz-se necessário, uma vez que a estabilidade me- cânica do fixador depende de diversos fatores inerentes ao aparelho. No tratamento de enfermidades músculoesqueléticas, as pesquisas em busca da melhoria do desempenho destes dispositivos e seu curso clínico são motivos de análise na literatura médica ${ }^{2-5}$. O incremento na estabilidade, modificando-se detalhes da disposição dos pinos, das articulações e das hastes, altera o desempenho do fixador, maximizando a rigidez do sistema, o que pode vir a aumentar a velocidade para atingir-se a consolidação óssea. Estes ajustes e alterações, no momento da montagem do aparelho, podem levar a resultados esperados e satisfatórios, facilitando, sobremaneira, o alcance do objetivo que é a consolidação óssea. O conhecimento destes princípios, que aumentam a eficácia dos fixadores externos, evidenciados pela pesquisa de base, trazem benefícios aos pacientes, que necessitam de tal recurso terapêutico.

Este estudo tem por objetivo evidenciar a resistência (rigidez) do sistema de fixação externa tubular

Trabalho realizado Programa de Pós-Graduação em Princípios da Cirurgia do Hospital Universitário Evangélico de Curitiba/ Faculdade Evangélica do Paraná, Curitiba, PR.

1. Médico Coordenador do Serviço de Ortopedia e Traumatologia do Hospital Sugisawa, Curitiba, PR, Brasil. 2. Professor Permanente do Programa de Pós-Graduação em Princípios da Cirurgia do Hospital Universitário Evangélico de Curitiba/Faculdade Evangélica do Paraná, Curitiba, PR, Brasil. 3. Médico do Grupo do Quadril do Serviço de Ortopedia e Traumatologia do Hospital Universitário Cajuru - Pontifícia Universidade Católica do Paraná , Curitiba, PR , Brasil. 4. Professora Titular de Engenharia Mecânica da PUC/PR. 5. Mestrando do Programa de Pós-Graduação em Princípios da Cirurgia do Hospital Universitário Evangélico de Curitiba/Faculdade Evangélica do Paraná, Curitiba, PR, Brasil. 
uniplanar unilateral, ao se modificar o número de barras longitudinais do fixador externo em um modelo sintético em traços estáveis e instáveis, submetido à uma força de compressão axial.

\section{MÉTODOS}

Este é um estudo experimental, realizado no Laboratório de Caracterização e Ensaios de Materiais do Parque Tecnológico da Pontifícia Universidade Católica do Paraná, Curitiba, PR, Brasil. Neste ensaio destrutivo foram analisadas 48 tíbias sintéticas seccionadas, estabilizadas com um fixador externo uniplanar, monolateral e com pinos de Schanz.

\section{MODELOS SINTÉTICOS DA TÍBIA COM FIXADOR EXTERNO}

\section{Modelo Sintético Poliuretano}

O modelo sintético mimetiza a tíbia direita humana, sendo manufaturado em poliuretano, polímero que compreende uma cadeia de unidades orgânicas, unidas por ligações uretânicas, amplamente usado em espumas rígidas e peças de plástico rígido. Cada modelo tem comprimento total de $39 \mathrm{~cm}$ e fenestra central, simulando um canal medular, de sete milímetros. Com serra elétrica em 24 tíbias sintéticas foi criado um corte com angulação de $15^{\circ}$ em relação ao eixo longitudinal do modelo em seu ponto médio. Em outros 24 modelos, realizou-se um corte com angulação de $45^{\circ}$ de inclinação em relação ao seu eixo longitudinal (Figura 1).

\section{Fixador Externo}

O fixador externo utilizado foi o Implantex ${ }^{\circledR}(\mathrm{Fi}-$ gura 2), na configuração uniplanar (linear), monolateral, com meios-pinos (half pin). Os pinos foram inseridos manu- almente, na face ântero-medial da tíbia sintética, na mesma disposição linear, através da perfuração prévia do seu trajeto, com perfurador à bateria Bosch ${ }^{\circledR}$ - Switzerland, modelo PSR 7,2 VE "electronic", a 400 rpm, utilizando-se uma broca de $3,5 \mathrm{~mm}$. Eles foram ancorados nas duas extremidades do modelo de poliuretano que mimetizaram as corticais ósseas. Foram conferidas, a angulação e a profundidade dos pinos de Schanz nos modelos, com o uso de um goniômetro e paquímetro digital de 150 milímetros de profundidade (Sylvac $\left.{ }^{\circledR}\right)$.

Os pinos foram inseridos com uma angulação de 90 graus em relação à diáfise, na cortical medial do modelo sintético. A profundidade de sua inserção era suficiente para romper a cortical oposta do ponto inicial de inserção, até $2 \mathrm{~mm}$, em todos os modelos. Os pinos utilizados foram os de Schanz, de aço 316 LVM, de $4.8 \mathrm{~mm}$, da marca Implantex ${ }^{\circledR}$, com extremidade rosqueada de $40 \mathrm{~mm}$, inseridos em número de quatro. Foram inseridos obedecendo-se à disposição linear e equidistante entre si. De cada lado da área de secção, que simulava o sítio da osteotomia diafisária, foi colocado um pino à distância de $2 \mathrm{~cm}$ e outro à $10 \mathrm{~cm}$. A montagem do fixador procedeu-se com a fixação dos pinos na haste longitudinal de suporte. Ela era canulada e apresentava $11 \mathrm{~mm}$ de diâmetro, sendo manufaturada em aço inoxidável e unida por meio de conectores, pino-haste, próprios do fixador externo Implantex ${ }^{\circledR}$. Esta montagem deixava separação de $5 \mathrm{~mm}$ entre os fragmentos, recriando um defeito ósseo ou lacuna no foco fraturário.

\section{Máquina de Testes Instron ${ }^{\circledast}$}

Os corpos de prova foram submetidos ao teste de compressão axial, pela máquina Universal de Ensaios Instron $8502^{\circledR}$ com carga axial progressiva. Esta máquina possuía uma célula de carga de até 100 kgf. Os modelos de prova foram adaptados à máquina de testes, pelas suas extremidades, por meio de um corte transversal, sendo ele o mais superficial possível. Isto foi realizado, a fim de meIhorar a adaptação do modelo sintético à maquina de en-

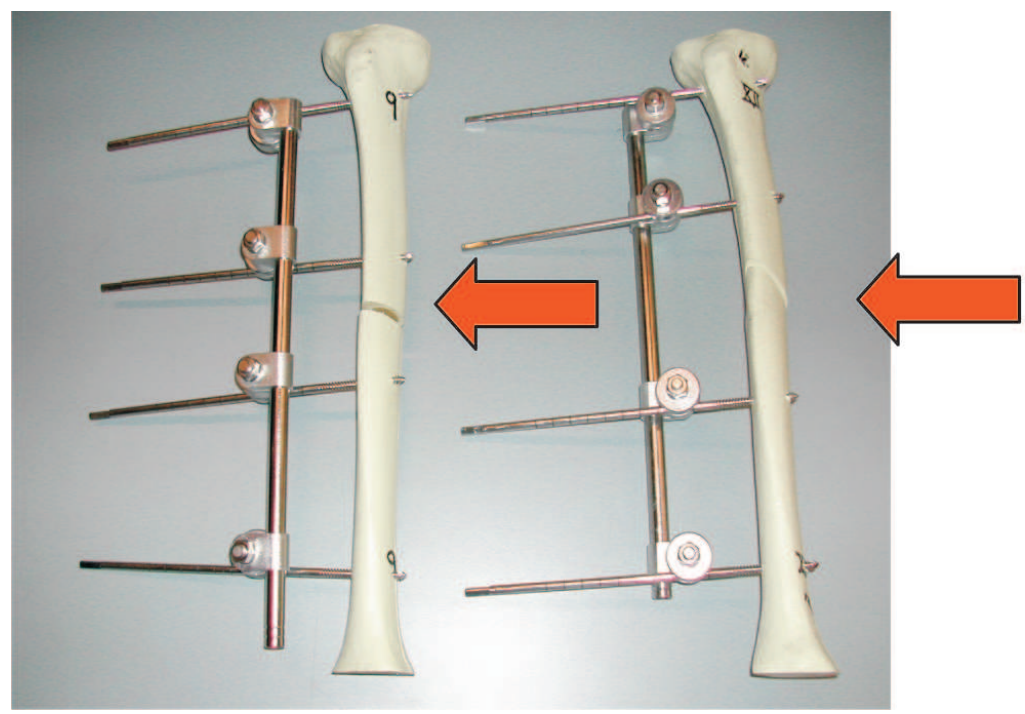

Setas indicando secção de 15 e 45 graus

Figura 1 - $\quad$ Aspecto da seção no modelo em poliuretano. 


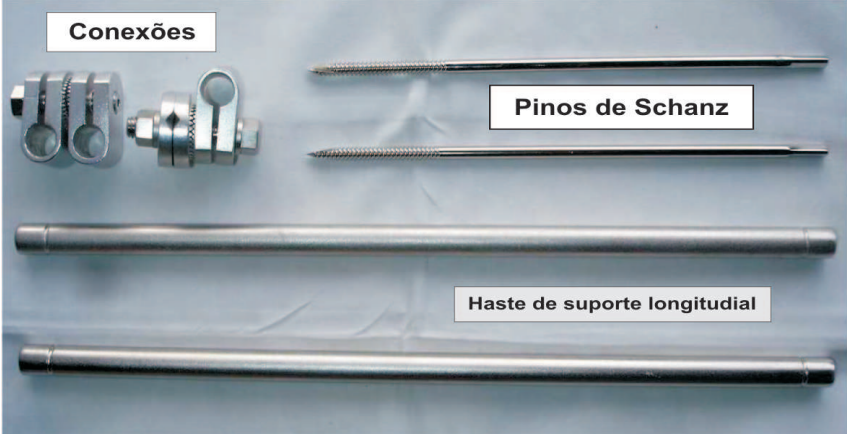

Figura 2 - Componentes do fixador externo Implantex ${ }^{\oplus}$.

saios, não permitindo o deslocamento do corpo de prova durante os testes (Figura 3).

\section{Delineamento Experimental}

Os 48 modelos foram divididos em quatro grupos de 12 corpos de prova cada um de acordo com o traço de fratura central e o número de barras (Figuras 4 e 5):

Grupo EU15 - Ângulo de 15\%(estável) e barra única.

Grupo ED15 - Ângulo de $15^{\circ}$ (estável) e barra dupla.

Grupo IU45 - Ângulo de $45^{\circ}$ (instável) e barra

única.

Grupo ID45 - Ângulo de $45^{\circ}$ (instável) e barra dupla.

\section{Ensaio mecânico}

O teste iniciava-se pela aplicação de uma de carga compressiva proporcionada pelo equipamento, que era controlada manualmente por meio de um botão giratório do painel digital da máquina de testes. A aplicação da carga de compressão axial era suspensa no momento do completo contato dos fragmentos, do modelo seccionado. Esta situação de contato completo era constatada, com o auxílio de um paquímetro digital (Figura 4). Os valores obtidos nos testes foram aferidos e anotados para posterior análise estatística. As unidades de medidas utilizadas foram a força em quilograma força e o deslocamento, em décimos de milímetros.

\section{ANÁLISE ESTATÍSTICA}

Os dados coletados foram, inicialmente, sumarizados para o cálculo de medidas estatísticas descritivas. O valor de referência para p foi de 0,05. Essas medidas foram obtidas para cada um dos grupos analisados, sendo elas: a média, o desvio-padrão, a mediana e o quartís, construindo-se tabelas e quadros para descrever o comportamento dos dados. Um gráfico do tipo Box Plot foi construído para ilustrar o comportamento da variável analisada em cada combinação dos fatores dos grupos estudados. Em seguida verificou-se a normalidade dos dados a partir do diagrama Normal Plot. Como a normalidade dos dados não foi comprovada, realizou-se o teste,

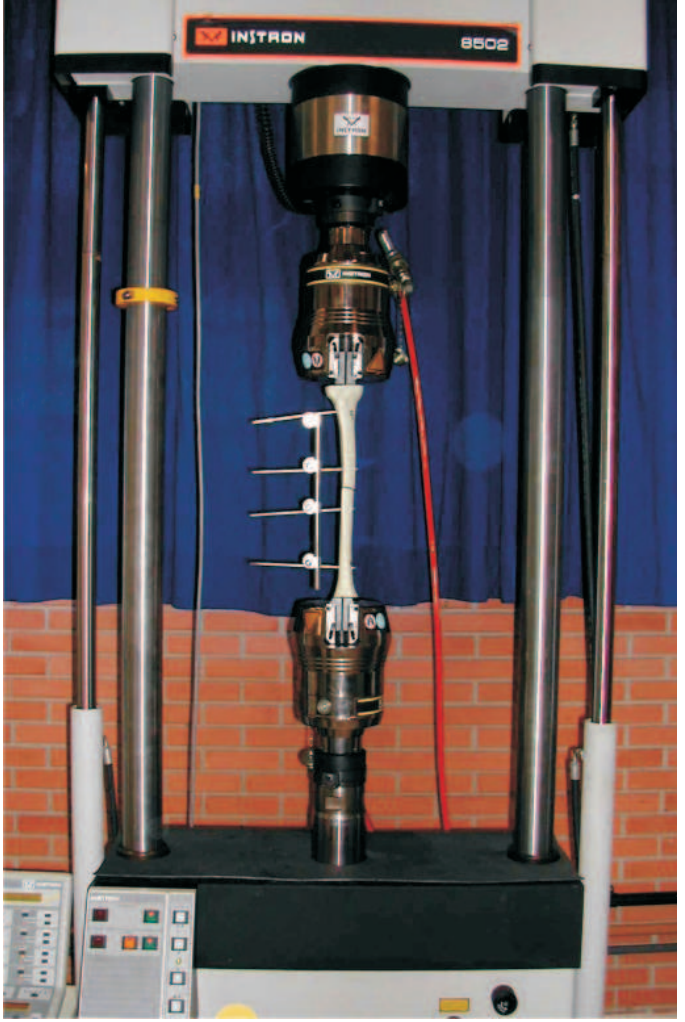

Figura 3 - Modelo de teste adaptado à máquina de testes Instron ${ }^{\circledR}$

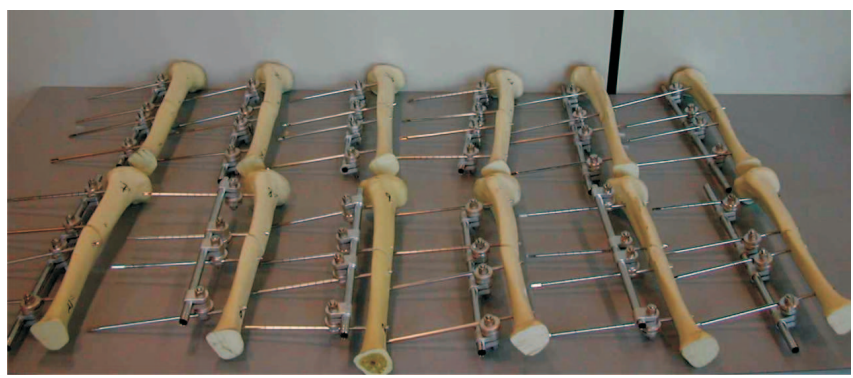

Figura 4 - Foto de um grupo de modelos com fixador e montagem barra única.

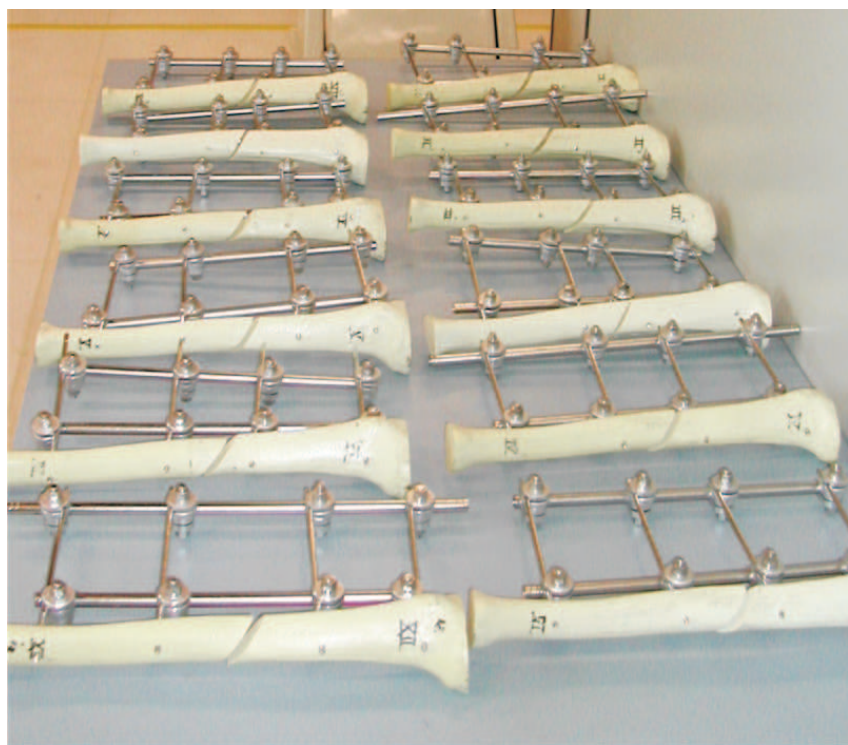

Figura 5 - Foto de um grupo de modelos com fixador e montagem barra dupla. 
não paramétrico, de Kruskall-Wallis, para comparar os quatros grupos e o teste DMS (diferença mínima significativa) para a identificação de diferenças significativas, caso houvesse.

\section{RESULTADOS}

\section{Resultado das Medidas}

Todos os 48 modelos foram testados seguindo as mesmas características e obtiveram os seguintes resultados. Não houve a falência das montagens dos modelos, durante os ensaios realizados, que inviabilizasse a aplicação da força compressiva até o contato das extremidades dos corpos de prova. Considerou-se a falência do sistema, como a quebra ou a falha do fixador externo nas regiões de conexões, entre as barras ou entre a barra e os pinos de Schanz, ou mesmo a quebra do modelo sintético. Não houve a soltura dos pinos de Schanz da interface osso-pino. Não se considerou a deformação plástica dos pinos até que o contato completo dos fragmentos fosse obtido. Observou-se que as respostas com barra dupla são bastante superiores às respostas com barra única. Observou-se ainda que o grupo barra única instável tem variabilidade muito menor que os demais grupos, ou seja, apresenta resultados mais homogêneos, além de ter apresentado a menor média. A figura 6 ilustra o comportamento dos fatores através da representação de suas respectivas médias.

Como os dados não apresentaram distribuição normal, aplicou-se um teste estatístico não paramétrico, o de Kruskal-Wallis, com valor de 35,26, associada a $p=0,000000107$, ou seja, existe diferença altamente significativa entre os quatro grupos analisados. O teste DMS, que complementa o teste Kruskal-Wallis, aponta a existência de diferença significativa entre os resultados obtidos na barra dupla e barra única, considerando-se nível de significância de 5\%.

\section{DISCUSSÃO}

A terapêutica ortopédica tem o objetivo da consolidação das fraturas, com a melhor qualidade possível e o mínimo de danos teciduais, indicando-se procedimentos que diminuam a morbidade e a mortalidade, ao abordarse tanto as lesões ósseas e como as musculares. Faz-se, assim, com que os pacientes retornem às atividades diárias, de maneira rápida e segura, otimizando os resultados finais das lesões do aparelho músculo-esquelético.

Vários autores revisam os conceitos básicos buscando o conhecimento das técnicas e características dos dispositivos ${ }^{2,46,7}$. A possibilidade de otimizar sua utilização, diminuindo as complicações e aumentando a resistência às forças que sobre os fixadores possam ser aplicadas, justificam a necessidade da melhoria dos conhecimentos das técnicas de implantação dos mesmos. Estas informações viabilizam a expansão e a difusão do conhecimento, no emprego dos fixadores externos à comunida-

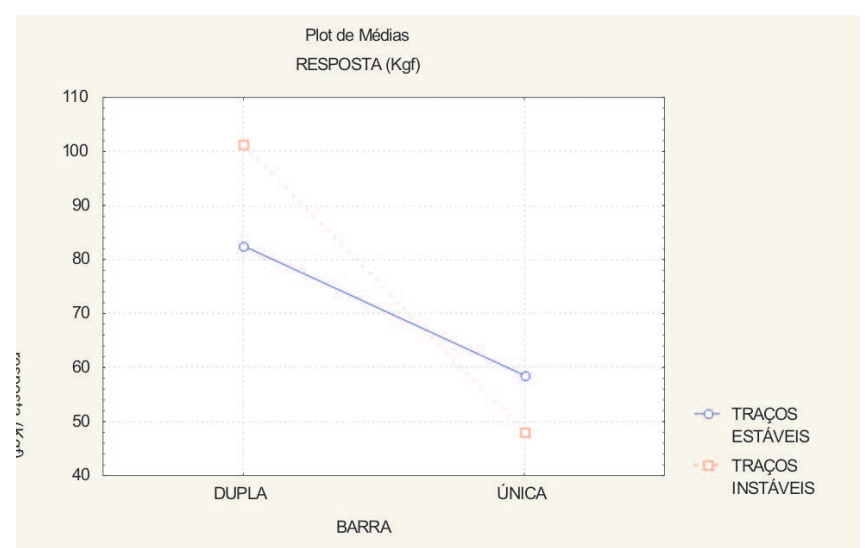

Figura 6 - Gráfico de comparação dos grupos de prova e força axial.

de científica. Este experimento utilizou configuração simples, quanto ao número de barras e de pinos de Schanz, usando uma única barra lisa e quatro pinos, conforme Dell'occa $^{8}$ postulou, na montagem de um sistema monoplanar. Neste estudo não houve a soltura dos pinos das barras, com superfície lisa.

A opção de utilizar-se uma barra de superfície recartilhada foi estudada por Mercadante et al. ${ }^{5}$ que, igualmente, observaram que a mesma não se soltava dos pinos de Schanz. A linearidade da configuração do fixador externo deve apresentar a simplicidade e a segurança da colocação dos pinos, na face ântero-medial da tíbia do ser humano, a qual não apresenta planos musculares e de feixes vásculo-nervosos, definidos como corredores de segurança. Estes corredores, quando respeitados, diminuem a dor do pós-operatório, a ocorrência de lesões iatrogênicas vásculo-nervosas e facilitam a aplicação dos pinos de Schanz, sem visualização direta do ponto de entrada do pino no osso (técnicas percutâneas).

As condições das partes moles e sua situação, influenciam a decisão na prática clínica, para a colocação dos pinos de Schanz nos ossos. Esta situação é crítica, quando frente à necessidade do tratamento de fraturas expostas que apresentam graves lesões dos tecidos, em decorrência do próprio trauma inicial. Em pacientes obesos, onde impreterivelmente a barra de conexão estará distante da superfície óssea, em decorrência do panículo adiposo espesso, a avaliação da referida distância, novamente tem sua relevância, pois ela alteraria a resistência do sistema de fixação, quando selecionado para o tratamento destes indivíduos.

O aumento do número de barras na montagem do fixador externo pode auxiliar no aumento da estabilidade $^{9,10}$. Este modelo experimental busca a comprovação da referida afirmação, usando um modelo sintético, simulando o osso humano. O traumatologista é capaz de realizar o planejamento pré-operatório, para a redução da fratura e a escolha do melhor posicionamento dos pinos, quando entende a anatomia local e a ação muscular. A estabilidade da fratura deve ser obtida com o auxílio de implantes que resistam às forças musculares 
deformadoras ${ }^{11}$ daquela região até a consolidação ${ }^{12}$, ou a conversão do método de fixação externa, a um método mais estável ao previamente utilizado ${ }^{13}$. Apuradas e precisas técnicas permitem que estes aparelhos possam ter maior capacidade de resistência à soltura e ao afrouxamento, gerando maior estabilidade do foco fraturário até a consolidação plena. Isto evita a conversão a outros implantes, o que diminui a morbidade dos indivíduos submetidos ao tratamento com os fixadores.

O conceito da estabilidade tem sua importância ao considerar-se a aposição dos fragmentos ósseos que, quando em posição anatômica, aumentam a estabilidade e propiciam a consolidação. Porém, esta situação ideal não é sempre possível na prática clínica, transferindo-se ao dispositivo de fixação externa a necessidade de conferir uma rigidez extra, necessária à cicatrização do tecido ósseo. A confecção de um corte em secção no modelo e a redução imperfeita das partes do modelo em estudo, em diástase, simulam situação real.

Os estudos clínicos, bem como os modelos experimentais de fixadores externos, comprovam a necessidade da compreensão do seu comportamento, sejam em condições ideais de experimentação ou de ensaios clínicos do uso de fixadores no tratamento de fraturas diafisárias da tíbia ${ }^{4,14,15}$. Do ponto de vista teórico, o experimento usa os princípios de momento de força ${ }^{16}$. O momento de força tem sua definição como uma força aplicada diretamente a um ponto fixo, em determinada distância, que promove tendência de giro deste ponto sobre um eixo. Quanto maior for a distância da aplicação da força dos pontos fixos, maior será a tendência de giro. No experimento, o momento de força se expressa pela estabilidade do sistema de fixação externa, ou seja, maior o momento de força, menor a estabilidade. Os modelos sintéticos, confeccionados em poliuretano, foram utilizados devido ao seu formato, similar à tíbia humana e às características mecânicas do material, semelhantes às condições humanas ${ }^{17}$, próprias para estudos de resistência, razão pela qual foi a opção neste experimento. A resistência do modelo confeccionado em poliuretano foi suficiente para a realização dos testes mecânicos, sendo que não houve a soltura dos pinos dos modelos em sua interface metalpoliuretano, ou a quebra do material. Observação similar foi feita por Mercadante et al. ${ }^{5}$ na avaliação da resistência entre o pino de Schanz e os corpos de prova, em modelos feitos de tubos de plástico, demonstrando que os modelos resistem às forças de deformação, necessárias aos testes de avaliação de estabilidade dos fixadores. O osso sintético em questão não contempla a presença da fíbula que contribui para a estabilidade do sistema, e representou uma limitação do estudo.

Analisando-se os dados estatísticos, pode-se observar variabilidade dos resultados das forças compressivas necessárias ao contato das secções dos modelos, apresentadas entre os grupos estáveis e instáveis. Observou-se que as respostas com barra dupla são bastante superiores às respostas com barra única. O grupo barra única instável apresentou variabilidade muito menor que os demais grupos, com resultados mais homogêneos, além de ter apresentado a menor média. Foi evidenciado a existência de diferença significativa entre os resultados obtidos na barra dupla e barra única, considerando nível de significância de $5 \%$. Os dados coletados neste experimento corroboram com a teoria do aumento da estabilidade dos fixadores externos, quando é adicionada uma barra de conexão à montagem.

A aplicação deste conhecimento na prática diária do tratamento das fraturas da tíbia otimiza o tratamento quando utilizado este dispositivo. O modelo experimental descrito deve ser considerado como um protótipo para outros estudos, a fim de melhor conhecer a mecânica dos dispositivos de fixação externa que são utilizados na prática médica.

Em conclusão, a montagem do fixador externo com uma haste longitudinal dupla é mais estável que as demais, quando submetidas à uma força de compressão axial.

\title{
A B S T R A C T
}

\begin{abstract}
Objective: This is an experimental study with the objective of analyses the increase of rigidity in synthetic tibia bones with external fixators, with single and double connecting rods and with stable and unstable fracture patterns. Methods: The external fixators were used in the monoplanar, half pin configuration submitted to an axial compression load using the connecting bar in different patterns. Forty-eight similar models to the human tibia had been used. In all the models were left an interval of 0,5 cm between the fragments and were made cuts of $15^{\circ}$ and $45^{\circ}$ to simulate stable and unstable fracture patterns, respectively. The models had been divided in four groups in accordance with the cut $\left(15^{\circ}\right.$ and $\left.45^{\circ}\right)$ and the number of metallic bars in assembly $(1$ and 2 bars). The study used the Instron ${ }^{\circledR}$ testing machine which the models were submitted to the axial load until the fragments that were separated by a gap made full contact. Results: The instability forces in the assembly with a double bar had been sufficiently superior to the forces with only one bar. It was still observed that the group with unstable fracture pattern and with only one connecting rod had a lesser variability compared with the other groups and presented more homogeneous results, with a minor average. Conclusion: The assembly with a double longitudinal connecting rod in the studied models is more stable than the others to stand compression load.
\end{abstract}

Key words: External fixators. Tibia. 


\section{REFERENCIAS}

1. Kenwright J, Harris JD, Evans M. External skeletal fixation for tibia shaft fractures. J Bone Joint Surg Br.1980; 62B(11):525.

2. Sisk DT. External fixation. historic review, advantages, disavantages, complications, and indicatons. Clin Orthop Relat Res.1983;180(11):15-22.

3. Sisk DT. General principles and techniques of external skeletal fixation. Clin Orthop Relat Res. 1983;180(11):96-100.

4. Ramos MRF, Rotbande IS, Shehata I, Knackfuss I. Contribuição ao estudo mecânico da fixador externo tubular AO. Rev Bras Ortop. 1999; 34(2):134-8

5. Mercadante MT, Kojima K, Hungria JOS, Zan RA, Abulasan T, lamaguchi RB, Mezzalira LG.Estudo mecânico comparativo de quatro montagens de fixador externo monolateral submetido à força de torção. Rev Bras Ortop. 2003; 38(3):106 -16.

6. Rotbande I, Ramos MRF. Atualização em fixação externa: conceitos e revisão. Rev Bras Ortop. 2000;35(4):103-8.

7. Ramos MRF, Rotbande IS, Shehata I, Knackfuss I. Estudo do perfil mecânico de fixador externos de plataforma. Rev Bras Ortop.1998;33(7):552-6.

8. Dell'occa AF. External fixation. In: Rüedi PT; Murphy WM. AO Principles of Fracture Management. New York: Thieme, 2000; 233-47.

9. Behrens F, Johnson W. Unilateral external fixation: methods to increase and reduce frame stiffness. Clin Orthop Relat Res.1989; 241(1): 48-56.

10. Matsushita T, Nakamura K, Ohnishi I, Kurokawa T. Sliding performance of unilateral external fixator for tibia. Med Eng Phys.1998; 20(1):66-9.

11. Draper ER, Strachan RK,Hughes SP, Nicol AC, Paul JP. The design and performance of an experimental external fixator with variable axial stiffness and a compressive force transducer. Med Eng Phys. 1997; 19(8):690-5.
12. Behrens F. General theory and principles of external fixation. Clin Orthop Relat Res. 1989; 241(1):15 -123.

13. Ming JH, Zhou PH, Zhou JP. Biomechanical analysis of fracture fixation with external fixator in vivo. Chin J Traumatol. 2006; 9(2):100-4.

14. Ramos MRF, Rotbande IS, Shehata I, Knackfuss I. Estudo do perfil mecânico de fixador externos de plataforma. Rev Bras Ortop.1998;33(7):552-6.

15. Ramos MRF, Rotbande IS, Shehata I. Estudo do comportamento mecânico de sistemas de fixação externa. Rev Bras Ortop. 1999;34(8):460-74.

16. Ramalho F, Nicolau GF, Toledo PAS. Os fundamentos da física 1.8 ed, Curitiba: Moderna, 2003; 361-3.

17. Szivek JA. Synthetic materials and structures used as models for bone. In: Yuehuei HA, Draughn RA. Mechanical testing of bone and the bone-implant interface. New York:CRC Press, 2000;15971.

Recebido em 15/01/2009

Aceito para publicação em 13/03/2009

Conflito de interesse: nenhum

Fonte de financiamento: nenhuma

\section{Como citar este artigo:}

Kume MH, Malafaia O, Dietz UA, Deeke M, Borsato KS, Menegotto FG, Pundek MRZ. A influência do número de barras na resistência à compressão axial dos fixadores externos monoplanares em modelos de tíbias de poliuretano. Rev Col Bras Cir. [periódico na Internet] 2010; 37(1). Disponível em URL: http://www.scielo.br/rcbc

\section{Endereço para correspondência:}

Marcio Hiroaki Kume

E-mail: ipem@evangelico.org.br 